\title{
Contexto da parceria como qualificador da gestão de projetos universidade-empresa
}

\author{
Eduardo Vicente Albertin ${ }^{\mathrm{a}, *}$, Daniel Capaldo Amaral ${ }^{\mathrm{b}}$ \\ a,*evalbert@sc.usp.br, EESC/USP, Brasil \\ bamaral@sc.usp.br, EESC/USP, Brasil
}

\begin{abstract}
Resumo
A literatura possui significativa quantidade de fatores críticos para o sucesso na gestão de projetos colaborativos, do tipo universidade-empresa. Esses fatores foram identificados por meio de estudos de casos múltiplos e levantamentos (surveys). Não há, portanto, estudos aprofundados verificando esses resultados. A pesquisa apresentada é do tipo observação participante em dois projetos, realizados entre um mesmo grupo de pesquisa e empresa. Avalia-se a percepção da equipe, a dos coordenadores dos projetos e do participante-observador (diário de campo), complementada pela análise de documentos e percepção dos stakeholders. 0 resultado mais significativo do caso é a indicação de que fatores relacionados ao contexto da parceria, tidos como críticos para o sucesso, agiram como condicionantes. Esse dado traz novas hipóteses sobre as listas de fator crítico e indica novas direções para a pesquisa.
\end{abstract}

Palavras-chave

Gerenciamento de projetos colaborativos universidade-empresa. Fatores críticos de sucesso. Desenvolvimento de produtos. Gestão de projetos.

\section{Introdução}

As empresas estão recorrendo à formação de alianças, parcerias, redes e outras formas de união para desenvolver e lançar produtos inovadores, com mais qualidade, velocidade e menor custo. Esses arranjos possibilitam a combinação de competências, divisão do investimento em pesquisa e desenvolvimento ( $P \& D)$ e compartilhamento de custos e riscos (AMATO NETO, 2000). Permitem também acesso a benefícios que a empresa não poderia obter por si própria (CAMARINHA-MATOS; AFSARMANESH, 2005; RYCROFT; KASH, 2004).

Os dados do levantamento nacional sobre inovação (PINTEC, 2003; 2005; 2007) indicam crescimento desse tipo de cooperação no Brasil, de $25,6 \%, 29,7 \%$, chegando a $31,4 \%$, respectivamente. Desenvolver ferramentas gerenciais, capazes de apoiar essa estratégia, é, portanto, fundamental.

Os métodos de gerenciamento de projetos são uma importante ferramenta para garantir a efetiva colaboração. Porto (2000) constatou que os empresários que optam por estabelecer relações com universidades concordam fortemente que a gestão de projetos é pré-requisito à colaboração. Plonski
(1999) e Barnes, Pashby e Gibbons (2006) apontam que a colaboração universidade-empresa (U-E) é um desafio gerencial complexo, desde o alinhamento da percepção da diferença cultural e dos objetivos dos parceiros, até a administração cotidiana dos projetos, o que reforçaria a necessidade de métodos avançados para garantir o controle eficiente de tais projetos.

Há autores que apontam um número significativo de projetos mal-sucedidos (CYERT; GOODMAN, 1997; BUSINESS AND HIGHER EDUCATION FORUM, 2001), outro indício da necessidade de aprimoramento nas técnicas gerenciais.

0 número de pesquisas que estudam a aplicação de ferramentas de gerenciamento de projetos, especificamente em projetos do tipo U-E, pode ser considerado pequeno, conforme a revisão bibliográfica deste artigo. Um dos esforços principais das pesquisas na área tem sido a identificação de fatores críticos de sucesso, específicos para esse tipo de projeto. Não há, porém, uma sistematização conclusiva sobre o assunto. 
0 trabalho compreende análise teórica e empírica sobre os fatores críticos de sucesso (FCSs) no gerenciamento de projetos de P\&D colaborativos $\mathrm{U}-\mathrm{E}$, em particular em dois projetos inseridos no contexto de um programa maior de pesquisa, conduzido entre uma empresa desenvolvedora de software e um grupo de pesquisa.

\section{Questão de pesquisa e objetivos}

Os FCSs consolidados na literatura para o gerenciamento de projetos colaborativos do tipo $\mathrm{U}$-E explicam os resultados de projetos semelhantes realizados no Brasil? Qual a influência desses fatores nos resultados dos projetos?

Essas são as questões de pesquisa de que trata este artigo. Os objetivos específicos, portanto, são:

- identificar na literatura os FCSs para gerenciamento de projetos colaborativos U-E;

- verificar a presença e avaliar a influência dos FCSs em projetos distintos de um programa de colaboração U-E;

- verificar a presença dos FCSs descritos na literatura nos projetos estudados;

- avaliar a influência da presença, ou ausência, dos FCSs nos resultados de cada projeto;e

- utilizando os resultados anteriores, verificar a suficiência da lista de FCSs encontrada na literatura.

\section{Gestão de projetos e colaboração universidade-empresa}

Define-se projeto como "um esforço temporário empreendido para criar um produto, serviço ou resultado único" $\left(\mathrm{PMBoK}^{\circledast} \ldots\right.$, 2004), ou seja, temporário por possuir começo e fim definidos e único por ser diferente de todos os similares feitos anteriormente. Um programa é um conjunto de projetos relacionados e gerenciados de modo coordenado para a obtenção de benefícios e controle que não estariam disponiveis se fossem gerenciados individualmente $\left(\mathrm{PMBoK}^{\circledR}\right.$..., 2004). Verzuh (2000) considera um projeto de sucesso quando o produto é entregue no prazo, dentro do orçamento e com alta qualidade.

Esses autores definem gerenciamento de projetos de maneira geral, criando teoria para qualquer tipo de projeto. Paralelamente, há extensa literatura sobre colaboração, que estuda especificamente projeto colaborativo e envolve outros aspectos além do gerenciamento. Emden, Calantone e Droge (2006) apresentam a revisão mais extensa e recente sobre colaboração em desenvolvimento de produtos. Outros autores também o fizeram (EMDEN; CALANTONE; DROGE, 2006 ; MATTESSICH; MONSEY, 1992; KATZ; MARTIN, 1997). Em comum, apresentam a definição de colaboração como um trabalho conjunto realizado para atingir resultados desejados.

Camarinha-Matos et al. (2006), porém, a definem de forma mais detalhada, como um processo de criação compartilhada, por meio do qual um grupo de entidades alcança as capacidades um do outro. Definem colaboração como:

"Um processo no qual entidades compartilham informações, recursos e responsabilidades para, juntamente, planejar, implementar e avaliar um programa de atividades para alcançar uma meta comum".

Em artigo dedicado a analisar a colaboração U-E sob a ótica da gestão, Plonski (1999) destaca quatro desafios: i) o compartilhamento de uma visão multidimensional, integrada e centrada no desenvolvimento de competências humanas; ii) a percepção clara das missões distintas, e complementares, da universidade e da empresa no processo de inovação; iii) o desenvolvimento de respostas inovativas às diversas necessidades de colaboração; e iv) capacitação para a gestão eficaz da colaboração U-E. Observe que, no último deles, o autor enfatiza a questão do desenvolvimento de novos métodos gerenciais.

Barnes, Pashby e Gibbons (2002) estudam especificamente a questão do gerenciamento na parceria. Eles reforçam a influência dos fatores culturais na colaboração U-E, divididos em prioridade relativa, perspectivas e horizontes de tempo. Eles sugerem que um balanceamento das necessidades da academia e da empresa seja alcançado para que a colaboração encontre o sucesso.

Segatto-Mendes (1996) descreve um modelo conceitual básico sobre colaboração U-E, o qual fornece as motivações para universidades e empresas se decidirem a trabalhar juntas, e também as barreiras e/ou facilitadores que atuam no processo de colaboração.

Portanto, conclui-se que a maioria dos autores abordam a colaboração U-E de maneira geral, como um tipo entre outros arranjos interorganizacionais, e que são unânimes em citar como diferencial desse tipo de colaboração o aspecto da diferença cultural entre os parceiros. Observa-se, também, que há poucos estudos mais específicos, isto é, identificando práticas e ferramentas gerenciais e voltados para a gestão de projetos. As exceções atêm-se na questão da identificação de fatores críticos de sucesso, que são descritos em detalhes na próxima seção. 


\section{Fatores críticos de sucesso (FCSs) na gestão de projetos colaborativos U-E}

A definição de fatores críticos de sucesso (FCSs) refere-se como o

número limitado de áreas nas quais resultados satisfatórios assegurarão desempenho competitivo de sucesso para indivíduos, departamentos ou organizações (ROCKART; BULLEM, 1981, p. 7).

Nessa pesquisa, FCSs são os fatores-chave, determinantes do desempenho em projetos colaborativos U-E.

Três estudos foram encontrados na literatura relativos à determinação de FCSs na gestão de projetos de P\&D colaborativos.

0 primeiro estudo foi o de Davenport, Davies e Grimes (1998) sobre a colaboração entre empresas e universidades, inseridos no Programa de Tecnologia para o Crescimento de Negócio (Technology for Business Grouth, TGB) apoiado pelo governo da Nova Zelândia. Os cinco primeiros FCSs classificados como de importância "vital" na colaboração constam do Quadro 1.

Embora quase todos os projetos concluídos tenham sido considerados como de sucesso, a maioria dos gerentes descreveu problemas, sendo os principais:

- metas irreais ou confusas;

- expectativas desalinhadas;

- falta de confiança;

- honestidade e abertura;

- agenda oculta;

- falta de compromisso;

- falta de comunicação; e

- desentendimentos entre parceiros.

Esses problemas consistem em quase a antítese dos FCSs mais bem classificados na pesquisa. Ao estudar o caso mais amplamente e ao longo do

Quadro 1. FCSs considerados de importância vital pelos participantes do o programa TGB.

\begin{tabular}{|c|c|}
\hline Classificação & $\begin{array}{l}\text { FCSs classificados como de } \\
\text { importância vital }\end{array}$ \\
\hline 1 & Respeito mútuo e confiança entre parceiros; \\
\hline 2 & $\begin{array}{l}\text { Compromisso da alta gerência em todas } \\
\text { as fases; }\end{array}$ \\
\hline 3 & $\begin{array}{l}\text { Compreensão clara das responsabilidades } \\
\text { e tarefas; }\end{array}$ \\
\hline 4 & $\begin{array}{l}\text { Seleção do parceiro “certo" de P\&D } \\
\text { colaborativo; }\end{array}$ \\
\hline 5 & Metas comuns sem agendas ocultas. \\
\hline
\end{tabular}

Fonte: Adaptado de Davenport, Davies e Grimes (1998). tempo, os autores perceberam a importância da diferença cultural e da confiança nos projetos. Concluíram, assim, que a confiança, goodwill trust, entre os parceiros foi o fator mais importante no gerenciamento de projetos $\mathrm{U}$-E, o qual pode ser desenvolvido quando ocorre a repetição de relacionamentos colaborativos.

No segundo estudo encontrado na literatura, Mora-Valentin; Montoro-Sanches e Gerra-Martin (2004) analisaram o impacto de uma série de FCSs em 800 acordos de colaboração entre empresas espanholas e organizações de pesquisa ocorrido entre 1995 e 2000. Dez FCSs foram selecionados da literatura e classificados em duas categorias: contextuais e organizacionais. Como resultado, os FCSs destacados para as empresas e para as organizações de pesquisa são apresentados no Quadro 2.

0 terceiro estudo encontrado na literatura foi desenvolvido por Barnes, Pashby e Gibbons (2006). Comparado aos dois estudos descritos anteriormente, considera maior número de FCSs e uma melhor organização, ao classificá-los em temas. Os temas e classificação são apresentados na Figura 1.

OsFCSs encontrados na literatura foram avaliados em seis estudos de caso - cinco deles ocorreram entre a Warwick Manufacturing Group (WMG) da School of Engineering da University of Warwick e $25 \mathrm{com}$ empresas da indústria automotiva. 0 sexto estudo de caso foi entre a WMG e uma empresa da indústria aeroespacial. 0 estudo revelou semelhança entre o gerenciamento dos FCSs que impactaram sobre a colaboração nos seis casos e sobre aqueles identificados nos estudos prévios de colaboração U-E. Os resultados encontrados por Barnes, Pashby e Gibbons (2006) reforçaram a hipótese de que os fatores identificados que influenciam a colaboração E-E também se aplicam ao caso da U-E.

\section{Método}

Esta pesquisa caracteriza-se como uma pesquisa qualitativa, segundo Godoy (1995), e o delineamento

Quadro 2. FCSs como resultado da pesquisa.

\begin{tabular}{|c|c|}
\hline Para as empresas & $\begin{array}{c}\text { Para as organizações } \\
\text { de pesquisa }\end{array}$ \\
\hline Compromisso & Ligação anterior \\
\hline Ligação anterior & Comunicação \\
\hline Definição de objetivos & Compromisso \\
\hline Conflitos & Confiança \\
\hline & Reputação dos parceiros \\
\hline
\end{tabular}

Fonte: Adaptado de Mora-Valentin, Montoro-Sanches e Gerra-Martin (2003) 
Questões da diferença cultural

Diferenças em prioridade/prazos

Publicação em domínio público

Falta de compreensão de imperativos de negócios (acadêmico)

Falta de flexibilidade (empresa)

Direitos de propriedade intelectual e confidencialidade
Avaliação do parceiro

- Compatibilidade de cultura/modo de operação

- Compreensão mútua

- Expertise e forças complementares

- Parceiros de colaboração no passado

- Pessoal de alta qualidade

- Importância estratégica

- Objetivos complementares

- Sem agendas ocultas

- Experiência colaborativa

Gerente de projeto

- Treinado em gestão de projetos

- Diplomacia

- Experiência em colaboração

- Experiência multifuncional
Preparação do projeto e execução

Gerenciamento do projeto

- Objetivos definidos claramente

- Responsabilidades definidas claramente

- Planos dos projetos acordados mutuamente

- Objetivos realistas

- Recursos adequados

- Milestones do projeto definidos

- Acordos colaborativos simples

- Monitoramento do progresso regular

- Comunicação eficaz

- Entregas dos colaboradores asseguradas

Garantia de igualdade

- Benefício mútuo

- lgualdade de poder/dependência

- Igualdade de contribuição

Influências externas

- Necessidades do mercado

- Estabilidade corporativa

$\quad$ Fatores Universais de Sucesso
- Confiança Mútua
- Compromisso
- Flexibilidade
- Aprendizado
- Continuidade de pessoal
- Boas relações pessoais/time de trabalho
- Campeão em Colaboração
- Liderança

Figura 1. FCSs no gerenciamento de projetos de pesquisa colaborativa U-E. Fonte: Adaptado de Barnes, Pashby e Gibbons (2006).

pode ser classificado como pesquisa de campo. Dane (1990) utiliza o termo geral "pesquisa de campo" para classificar uma pesquisa que inclui a observação direta de eventos que ocorrem naturalmente. A autora indica também o rótulo “observação participante" quando o pesquisador participa do evento observado.

Gil (1999) aponta que a obsevação participantese desenvolve a partir da interação entre pesquisadores e membros das situações investigadas. Gold (1969 apud DANE, 1990, p. 159) definiu que a pesquisa é classificada como "participante-observador", quando o pesquisador participa das atividades e seu status como pesquisador é conhecido dos participantes.
Neste trabalho adota-se a classificação de Dane (1990), ou seja, pesquisa de campo, do tipo participante-observador, por esclarecer e delimitar a relação entre o pesquisador e o objeto de estudo. Justifica-se pelo fato de o pesquisador ter participado dos projetos colaborativos U-E em estudo, quando assumiu parte das atividades de gerenciamento. 0 papel duplo, pesquisador e profissional, era conhecido de todos os participantes.

\subsection{Unidade de análise}

A unidade de análise é o projeto de pesquisa. Analisam-se dois projetos, denominados A e B, realizados entre novembro de 2006 a outubro 
de 2007, período que corresponde à atuação do pesquisador nos projetos. Ambos faziam parte de um mesmo programa, que será denominado U-EA.

\subsubsection{Programa U-EA}

O Programa U-EA teve o objetivo de desenvolver um conjunto coeso de tecnologias de informática e métodos gerenciais. Foi composto de sete projetos com tamanho e nível de inovação distintos. Dois deles, denominados de A e B, são os selecionados como objeto de estudo para a pesquisa. Os critérios para a seleção foram:

- Tratar-se de um projeto colaborativo, de acordo com a definição de Camarinha-Matos et al. (2006) adotada no referencial teórico;

- Ter ocorrido no período coberto pela pesquisa (ou seja, de nov./06 a out./07 - durante o período de participação do autor dessa pesquisa como observador); e

- Possuir participantes das duas organizações (empresa e grupo de pesquisa) na equipe de projeto, além dos coordenadores do programa.

\subsubsection{Perfil das equipes de projeto}

Nos dois projetos pesquisados, participaram treze pesquisadores. Um grupo de oito permaneceu durante todo o decorrer dos projetos. Esse grupo foi escolhido para fazer parte do levantamento de campo sobre a percepção dos envolvidos. Os papéis de cada um deles, classificados segundo a definição de PMBoK ${ }^{\circledR}$ (2004), são apresentados no Quadro 3.

\subsection{Etapas}

A pesquisa partiu de uma revisão bibliográfica paralela a uma pesquisa de campo, iniciada com a inclusão do pesquisador na equipe de projeto. A Figura 2 apresenta o fluxograma das etapas da pesquisa.

A pesquisa de campo ocorreu com a observação direta do pesquisador sobre os eventos dos projetos $\mathrm{A}$ e $\mathrm{B}$, dos quais fazia parte. Os problemas eram discutidos com os demais participantes das equipes e anotados em um diário, que cobriu o período de novembro de 2006 até outubro de 2007. As

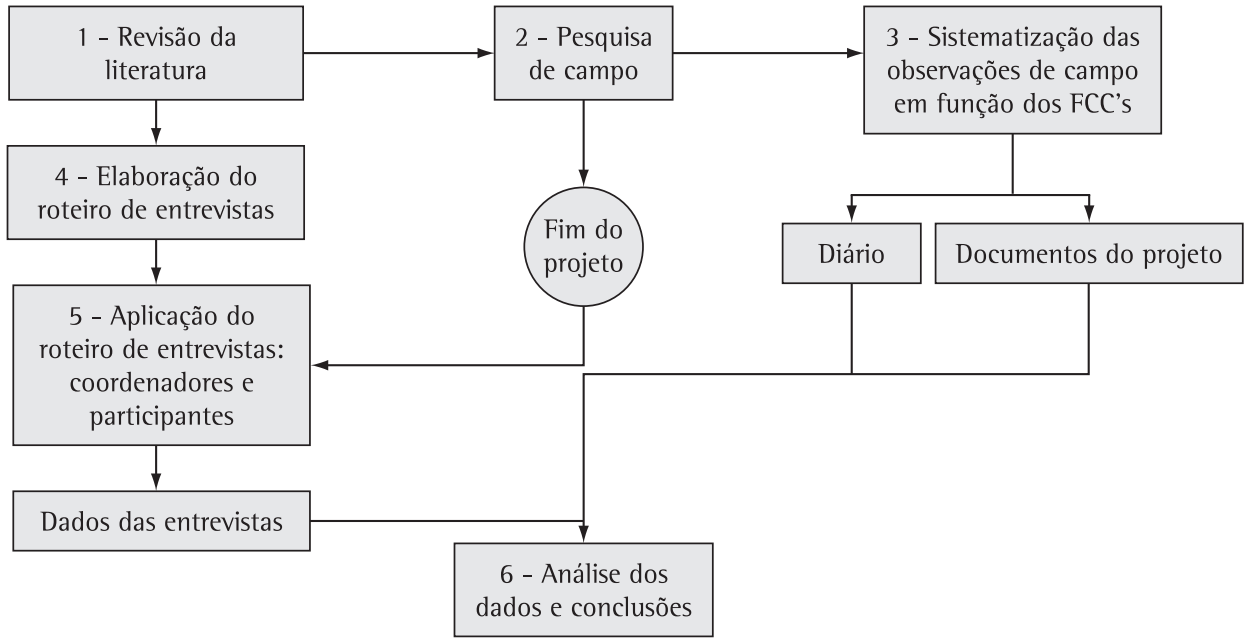

Figura 2. Fluxograma das atividades da pesquisa tema gerenciamento de projeto no projeto A.

Quadro 3. Papéis e participações no programa U-EA.

\begin{tabular}{|c|c|c|c|c|c|c|c|}
\hline \multicolumn{8}{|c|}{ Participantes do Programa U-EA } \\
\hline \multirow[b]{2}{*}{ Entidade } & \multirow{2}{*}{$\begin{array}{c}\text { Coordenadores do } \\
\text { programa (gerente } \\
\text { de programa e } \\
\text { stakeholders) } \\
\text { U-EA }\end{array}$} & \multicolumn{3}{|c|}{ Projeto A } & \multicolumn{3}{|c|}{ Projeto B } \\
\hline & & Coordenador & $\begin{array}{l}\text { Gerente } \\
\text { de } \\
\text { projeto }\end{array}$ & $\begin{array}{l}\text { Desenvolvedores } \\
\text { (membro da } \\
\text { equipe de projeto) }\end{array}$ & Coordenador & $\begin{array}{l}\text { Gerente } \\
\text { de } \\
\text { projeto }\end{array}$ & $\begin{array}{l}\text { Desenvolvedores } \\
\text { (membro da } \\
\text { equipe de } \\
\text { projeto) }\end{array}$ \\
\hline Universidade & 2 & 1 & $9^{*}$ & $8,6,5,3$ & & $9^{*}$ & 8,3 \\
\hline Empresa $\mathrm{A}$ & 7 & - & & 3 & 3 & - & 4 \\
\hline
\end{tabular}

1-9 - Cada número indica um participante. Esse código é utilizado na descrição dos resultados das entrevistas. $\left(^{*}\right)$ - Pesquisador: Participante-observador gerente de projetos. 
observações do diário de campo foram sistematizadas de forma a ter-se o maior nível de detalhamento possível, e depois organizadas em padrões lógicos e não cronológicos.

As entrevistas foram dirigidas a dois grupos de participantes. 0 primeiro formado pelos dois coordenadores do Programa U-EA, representantes da universidade e da empresa. No segundo grupo, as entrevistas foram dirigidas aos participantes da equipe responsável pela execução dos projetos e aos coordenadores dos projetos A e B.

0 roteiro de entrevista deste segundo grupo foi dividido em duas partes. A parte 1 continha 40 perguntas referentes à verificação da presença e à avaliação da influência dos FCSs consolidados na literatura, agrupados por temas. Uma pergunta para cada FCS, conforme Quadro 4. Essa lista pode ser considerada uma adaptação do trabalho de Barnes, Pashby e Gibbons (2006), pois o teve como ponto de partida, aprimorando-o com pequenas adaptações, conforme propostas de outros autores identificados na revisão.

A parte 11 continha perguntas abertas, solicitando a identificação de possíveis FCSs, percebidos no projeto e não pertencentes à lista da seção anterior. Além de o entrevistado expressar sua percepção sobre FCSs específicos, identificados na literatura, abriu-se a oportunidade de identificar FCSs não presentes.

0 roteiro de entrevistas dirigido aos coordenadores, primeiro grupo, diferiu na quantidade de perguntas. Além das duas seções já descritas, incluiu uma terceira parte com questões referentes à percepção dos resultados do projeto. 0 parâmetro de sucesso para o projeto foi o cumprimento das metas previstas em seu início, conforme definição de Verzuh (2000), descrita na seção 2.

Essa parte foi excluída dos membros da equipe porque se julgou que eles não teriam elementos para análise global dos resultados, por faltar-lhes a compreensão das relações com os vários projetos inseridos no Programa U-EA.

A versão final do roteiro de entrevistas foi definida após sua aplicação ter sido testada com um indivíduo interno, participante do Programa U-EA, e mais dois externos. 0 roteiro de entrevistas foi baseado nos FCSs classificados em temas, conforme Quadro 4.

Os resultados das entrevistas foram analisados, separadamente, por projeto e de acordo com as partes 1,11 e 111 do roteiro. Os resultados da parte 1 foram transformados em gráficos de barras, agrupados segundo temas de FCSs. Para cada tema de FCS foram criados dois gráficos, um sobre a
Quadro 4. Fatores críticos de sucesso utilizados na pesquisa.

\begin{tabular}{|c|c|}
\hline Temas & Fatores críticos de sucesso \\
\hline \multirow{10}{*}{$\begin{array}{l}\text { 1. Gerenciamento } \\
\text { do projeto }\end{array}$} & 1 Objetivos definidos claramente \\
\hline & 2 Objetivos conhecidos e aceitos \\
\hline & 3 Objetivos realistas \\
\hline & $\begin{array}{l}4 \text { Responsabilidades definidas } \\
\text { claramente }\end{array}$ \\
\hline & $\begin{array}{l}5 \text { Planos do projeto acordados } \\
\text { mutuamente }\end{array}$ \\
\hline & 6 Recursos adequados \\
\hline & 7 Milestones do projeto definidos \\
\hline & $\begin{array}{l}8 \text { Monitoramento regular do } \\
\text { progresso }\end{array}$ \\
\hline & 9 Comunicação eficaz \\
\hline & 10 Plano de gerenciamento de risco \\
\hline \multirow{7}{*}{$\begin{array}{l}\text { 2. Aspectos } \\
\text { gerais }\end{array}$} & 11 Confiança mútua \\
\hline & 12 Comprometimento \\
\hline & 13 Aprendizado - captação \\
\hline & 14 Aprendizado - utilização \\
\hline & 15 Continuidade de pessoal \\
\hline & $\begin{array}{l}16 \text { Boas relações pessoais entre os } \\
\text { parceiros }\end{array}$ \\
\hline & 17 Campeão em colaboração \\
\hline \multirow[t]{5}{*}{$\begin{array}{l}\text { 3. Gerente } \\
\text { de projeto }\end{array}$} & $\begin{array}{l}18 \text { Experiência em gerenciamento de } \\
\text { projetos }\end{array}$ \\
\hline & 19 Negociação \\
\hline & 20 Experiência em colaboração \\
\hline & 21 Experiência multifuncional \\
\hline & 22 Conhecimento técnico \\
\hline \multirow{9}{*}{$\begin{array}{l}\text { 4. Avaliação } \\
\text { do parceiro }\end{array}$} & 23 Compatibilidade de cultura \\
\hline & 24 Compreensão mútua \\
\hline & 25 Expertise complementar \\
\hline & 26 Experiência em colaboração \\
\hline & $\begin{array}{l}27 \text { Parceiros em colaboração no } \\
\text { passado }\end{array}$ \\
\hline & 28 Pessoal de alta qualidade \\
\hline & 29 lmportância estratégica \\
\hline & 30 Objetivos complementares \\
\hline & 31 Agenda oculta \\
\hline \multirow{4}{*}{$\begin{array}{l}\text { 5. Questões de } \\
\text { diferença } \\
\text { cultural }\end{array}$} & 32 lgualdade em prioridades e prazos \\
\hline & $\begin{array}{l}33 \text { Compreensão de imperativos de } \\
\text { negócios - academia }\end{array}$ \\
\hline & 34 Flexibilidade - empresa \\
\hline & $\begin{array}{l}35 \text { Direitos de publicação, } \\
\text { propriedade intelectual e } \\
\text { confidencialidade }\end{array}$ \\
\hline \multirow{3}{*}{$\begin{array}{l}\text { 6. Garantia de } \\
\text { igualdade }\end{array}$} & 36 Benefício mútuo \\
\hline & 37 lgualdade de poder \\
\hline & 38 lgualdade de contribuição \\
\hline \multirow{2}{*}{$\begin{array}{l}\text { 7. Influências } \\
\text { externas }\end{array}$} & 39 Necessidades do mercado \\
\hline & 40 Estabilidade corporativa \\
\hline
\end{tabular}

Fonte: Adaptado de Barnes, Pashby e Gibbons (2006). 
presença e outro sobre a influência nos resultados. Em seguida, classificou-se cada FCS como presente ou ausente utilizando-se esses resultados. As demais partes, formadas por questões abertas, tiveram as respostas codificadas e classificadas a posteriori.

\section{Apresentação e análise dos resultados}

De fato, as respostas concentram-se em torno de um FCS que já havia sido perguntado na entrevista: o FCS conhecimento técnico (22) do gerente de projeto.

\subsection{Projeto $A$}

\subsubsection{FCSs na percepção da equipe de projeto}

0 primeiro passo da análise foi identificar a percepção dos participantes do projeto quanto à presença e influência dos FCSs, a partir das questões fechadas, com escalas. Cada participante avaliou a presença e o nível de influência do FCS. As respostas foram sumarizadas em dois gráficos para cada tema do quadro 4: um para a presença e outro para a influência. Um exemplo para os FCSs do tema gerenciamento de projetos é apresentado na Tabela 1.

Cada item do gráfico, numerado na linha, representa um FCS. As barras representam a frequência de respostas para cada nível. Quanto à presença, havia dois níveis. A presença, sim, e a ausência, não. Quanto à influência, empregaram-se três níveis: não influente (não), influente (sim) e fortemente influente (FI).
A classificação da presença ou ausência foi feita pela observação da predominância maior ou igual a $75 \%$ das respostas afirmativas para um dos níveis. Quando houve predominância maior ou igual a 75\% da classe "não sei", o FCS foi considerado conflitante.

Na dimensão influência, a análise foi feita pela observação da quantidade de respostas afirmativas para as classes "não influente", "influente" e "fortemente influente". Assim, foram consideradas as respostas em função da maioria, sendo o grau de influência (influente ou fortemente influente) considerado conjuntamente na análise, pois, durante a aplicação, percebeu-se dificuldade, por parte dos entrevistados, em estabelecer diferenças entre os graus fortemente influente e influente (que não havia aparecido durante o teste do questionário).

Utilizando-se o exemplo da Tabela 1, como ilustração da análise, consideraram-se os FCSs relacionados ao tema gerenciamento de projetos, cuja presença, na percepção dos participantes, foi considerada inconclusiva para todos os FCSs. Isso significa que não houve uma opinião prevalecente no grupo. Com relação à influência desses fatores, todos foram considerados influentes, com exceção do fator plano de gerenciamento de risco (10), considerado conflitante.

Replicou-se esse procedimento para cada um dos temas em que foram agrupados os FCS (aspectos gerais, gerente de projeto, avaliação do parceiro, questões da diferença cultural, garantia de igualdade e influências externas), obtendo-se 6 pares de gráficos para o projeto $\mathrm{A}$. 0 conjunto completo dos dados pode ser consultado em Albertin (2008). Após a análise, cada FCS foi classificado de acordo com os critérios estabelecidos, e os resultados resumidos no Quadro 5.

Tabela 1. Presença e influência do FCS para o tema gerenciamento de projeto no projeto A. Verificação da presença dos FCSs e avaliação da influência dos FCSs (gerenciamento do projeto).

\begin{tabular}{|c|c|c|c|c|c|c|}
\hline \multirow[t]{2}{*}{ Objetivos } & \multicolumn{3}{|c|}{ Verificação } & \multicolumn{3}{|c|}{ Avaliação } \\
\hline & $\mathrm{T}(\operatorname{sim})$ & $\mathrm{T}$ (não) & $T$ (n.s.) & $T$ (f.i.) & $\mathrm{T}(\operatorname{sim})$ & T (não) \\
\hline Planos de gerenciamentos de riscos & 1 & 4 & 2 & 0 & 2 & 3 \\
\hline Comunicação eficaz & 3 & 3 & 1 & 4 & 2 & 0 \\
\hline Monitoramento regular do progresso & 5 & 1 & 1 & 5 & 1 & 0 \\
\hline "Milestones" dos projetos definidos & 1 & 5 & 1 & 1 & 4 & 1 \\
\hline Recursos adequados & 4 & 2 & 1 & 5 & 0 & 0 \\
\hline Planos de projetos acordados manualmente & 2 & 2 & 3 & 2 & 1 & 1 \\
\hline Responsabilidades definidas claramente & 4 & 2 & 1 & 3 & 3 & 0 \\
\hline Objetivos realistas & 2 & 2 & 3 & 4 & 2 & 0 \\
\hline Objetivos conhecidos e aceitos & 3 & 3 & 1 & 4 & 2 & 0 \\
\hline Objetivos definidos claramente & 4 & 3 & 0 & 3 & 2 & 0 \\
\hline
\end{tabular}

T (sim): Total de sim; T (não): Total de não; T (n.s.): Total não sabem; T (f.i.): Total fortemente influente. 
Quadro 5. Resultados compilados da percepção dos respondentes sobre os FCSs considerados presentes e influentes para o projeto A.

\begin{tabular}{|c|c|}
\hline \multicolumn{1}{|c|}{ Temas } & FCSs presentes - influentes \\
\hline $\begin{array}{l}\text { 4. Avaliação do } \\
\text { parceiro }\end{array}$ & $\begin{array}{l}25 \text { Expertise complementar } \\
30 \text { Objetivos complementares }\end{array}$ \\
\hline $\begin{array}{l}\text { 5. Questões da } \\
\text { diferença } \\
\text { cultural }\end{array}$ & $\begin{array}{c}33 \text { Compreensão de imperativos de } \\
\text { negócios - academia }\end{array}$ \\
\hline $\begin{array}{l}\text { 6. Garantia de } \\
\text { igualdade }\end{array}$ & 38 lgualdade de contribuição \\
\hline
\end{tabular}

Portanto, dos quarenta FCSs pesquisados no projeto $A$, quatro foram considerados como presentes e influentes. Apenas dois FCSs foram identificados como ausentes e influentes, isto é, não estavam presentes no projeto $\mathrm{A}$ : aprendizado - utilização (14) e igualdade de poder (37). A falta desses fatores afetou resultados, indicando possiveis prejuízos.

\subsubsection{Análise da percepção dos participantes quanto à existência de outros FCSs}

$\mathrm{Na}$ questão aberta do roteiro, o respondente teve a oportunidade para expressar-se de forma livre sobre outros fatores que poderiam ter contribuído para sucesso ou insucesso. 0 resultado é apresentado no Quadro 6.

\subsubsection{Comentários dos participantes do projeto A sobre os FCSs}

Alguns dos entrevistados expuseram suas percepções sobre os fatores críticos questionados, e a compilação das mais relevantes é descrita a seguir. Sobre os objetivos do projeto, um respondente afirmou que "não foram claramente definidos desde o início do projeto, e sim ao longo do tempo" e outro citou que "após uma reunião geral em dezembro de 2006, a visão dos objetivos começou a clarear”. Um dos respondentes citou que isso "deveu-se ao fato de não estarem definidos os requisitos do projeto".

\subsubsection{FCSs na percepção do participante-observador}

As observações mais relevantes registradas no diário de campo durante o período desta pesquisa foram compiladas e classificadas de acordo com a lista de FCSs de Barnes, Pashby e Gibbons (2006) e são apresentadas a seguir:

- Objetivos definidos claramente: em novembro e dezembro de 2006, em reuniões realizadas com a equipe para discussão das tarefas em andamento, os participantes declararam que tinham muitas
Quadro 6. FCSs percebidos pelos respondentes para o projeto $A$, segundo questão aberta.

\begin{tabular}{|c|c|}
\hline Respondentes & FCSs percebidos e não perguntados \\
\hline 1 & $\begin{array}{l}\text { Ter, em tempo integral, um gerente de } \\
\text { projeto com conhecimento técnico; }\end{array}$ \\
\hline 2 & $\begin{array}{l}\text { Definição clara dos requisitos no início } \\
\text { do projeto e sua manutenção ao longo } \\
\text { do tempo; }\end{array}$ \\
\hline 3 & Ter gerente técnico; \\
\hline 5 & $\begin{array}{l}\text { Ter especialista em desenvolvimento de } \\
\text { software web para definição de requistos } \\
\text { no início do projeto; }\end{array}$ \\
\hline 6 & Ter gerente técnico; \\
\hline 8 & $\begin{array}{l}\text { Ter gerente técnico especialista em } \\
\text { desenvolvimento de sistemas web; }\end{array}$ \\
\hline
\end{tabular}

dúvidas sobre os objetivos do projeto. Em meados de dezembro de 2006, houve uma reunião geral do coordenador do Programa U-EA, do parceirouniversidade, com os participantes de todos os projetos, quando foi feita uma apresentação esquemática do Programa U-EA e, desde então, e ao longo do tempo, os objetivos passaram a ser mais bem entendidos.

- Responsabilidades definidas claramente: participantes da equipe, ao serem indagados pelo gerente de projeto sobre suas responsabilidades, afirmaram não conhecer totalmente suas responsabilidades ou mesmo as implicações de suas ações no andamento do trabalho dos companheiros do projeto. Esse fato pode ser explicado devido ao desconhecimento dos seus papéis individuais

- Recursos adequados: foi verificado que havia a dificuldade de encontrar estudantes ou profissionais com qualificação específica para atuação no projeto dispostos a trabalhar pelos valores de bolsas de estudo, menores que os oferecidos pelas empresas, no mercado de trabalho.

- Comunicação eficaz: as reuniões mensais com a equipe completa do Programa U-EA ocorreram, sistematicamente, de dezembro de 2006 até os cinco primeiros meses de 2007. Após esse período, as reuniões passaram a ser separadas por projeto e coordenadas pelos respectivos responsáveis. 0 foco migrou para os esforços de gerenciamento e comunicação entre os coordenadores e a equipe e entre os participantes da equipe desenvolvedora, favorecendo a eficácia da comunicação.

- Comprometimento: todos os participantes da equipe disseram ter comprometimento com o projeto. No entanto, foi possível observar outras prioridades em parte dos membros, tais como: curso de graduação, mestrado, doutorado, aulas, outros trabalhos etc. Isso impactou no desempenho individual dos participantes. 
- Continuidade de pessoal: foi observado sobre três aspectos: rotatividade, faltas e horário de trabalho. Os motivos apontados foram:

- Rotatividade - Estágio em empresa, obtenção de bolsa de outra instituição, conclusão de curso, motivos pessoais ou outra atividade paralela;

- Assiduidade - A execução de tarefas do projeto foi prejudicada devido a faltas por motivos diversos como problema de saúde, viagem, provas, congressos, escrever artigos, escrever dissertação etc.;

- Dedicação dos participantes - A dedicação dos participantes dos projetos, graduandos e pós-graduandos, variou ao longo dos projetos. A atenção e entusiasmo do início não se mantiveram ao longo do projeto;e

- Variação do horário de trabalho - Os horários de trabalho de membros das equipes dos projetos eram diferentes e variáveis ao longo da semana.

- Conhecimento técnico do gerente de projeto: o projeto não contou com um responsável técnico conhecedor de todas as tecnologias envolvidas, em tempo integral, ou seja, uma pessoa envolvida com todos os projetos e na coordenação do Programa U-EA como um todo.

\subsubsection{Percepção dos coordenadores quanto aos resultados do projeto $A$}

As respostas da parte 111 do roteiro de entrevistas, aplicadas aos coordenadores do Programa U-EA e gerentes de projeto, são mostradas a seguir. Obtiveram-se respostas afirmativas de três, entre quatro respondentes, para as seguintes dimensões: contribuição ao apoio continuado do programa de pesquisa; envolvimento de projetos de estudantes; e recrutamento de estudantes. Obtiveram-se respostas negativas para três, dentre quatro respondentes, para as dimensões: benefícios financeiros e inovação tecnológica.

$\mathrm{Na}$ avaliação geral, três de quatro respondentes não consideraram o projeto A como um caso de sucesso. 0 respondente que não se manifestou justificou estar distante do projeto. Ele não se sentiu seguro em manifestar opinião e, como conclusão, o projeto A foi considerado como um caso de não sucesso.

\subsection{Projeto $B$}

\subsubsection{Análise da percepção dos participantes da equipe quanto aos FCSs da literatura}

As respostas das entrevistas para o projeto B, com relação aos FCSs, foram realizadas de maneira idêntica às do projeto A. Os dados de campo foram compilados em gráficos para cada tema. Os dados completos estão publicados em Albertin (2008). Sintetizando-se os resultados da análise dos quarenta FCSs pesquisados no projeto $\mathrm{B}$, treze foram considerados como presentes e influentes, na percepção dos respondentes, conforme apresentado no Quadro 7. Houve, nesse projeto, o caso de um FCS considerado ausente e influente, o FCS aprendizado - utilização (14).

\subsubsection{Análise da percepção dos participantes quanto à existência de outros FCSs}

A parte 11 do roteiro de entrevistas, com uma questão aberta para o respondente identificar potenciais fatores que não haviam sido questionados (e, portanto, não consolidados na literatura), não teve resposta dos entrevistados.

\subsubsection{Comentários dos participantes do projeto B sobre os FCSs}

Os comentários reforçaram uma avaliação positiva do projeto e a facilidade devido à menor duração. Uma resposta típica foi "o projeto B foi de curta duração, sua equipe era pequena e sabia-se onde chegar". Houve também respostas sobre o fato de o resultado ter sido menor que o

Quadro 7. Resultados compilados da percepção dos respondentes sobre os FCSs considerados presentes e influentes para o projeto $\mathrm{B}$.

\begin{tabular}{|c|c|}
\hline Temas & $\begin{array}{c}\text { Fatores críticos de sucesso } \\
\text { presentes - influentes }\end{array}$ \\
\hline $\begin{array}{l}\text { 1. Gerenciamento do } \\
\text { projeto }\end{array}$ & $\begin{array}{l}2 \text { Objetivos conhecidos e aceitos } \\
3 \text { Objetivos realistas } \\
4 \text { Responsabilidades definidas } \\
\text { claramente } \\
5 \text { Planos do projeto acordados } \\
\text { mutuamente } \\
6 \text { Recursos adequados } \\
8 \text { Monitoramento regular do } \\
\text { progresso }\end{array}$ \\
\hline 2. Aspectos gerais & $\begin{array}{l}12 \text { Comprometimento } \\
15 \text { Continuidade de pessoal } \\
16 \text { Boas relações pessoais entre os } \\
\text { parceiros } \\
17 \text { Campeão em colaboração }\end{array}$ \\
\hline 3. Gerente de projeto & 19 Negociação \\
\hline $\begin{array}{l}\text { 4. Avaliação do } \\
\text { parceiro }\end{array}$ & 29 Importância estratégica \\
\hline $\begin{array}{l}\text { 5. Questões da } \\
\text { diferença cultural }\end{array}$ & 34 Flexibilidade - empresa \\
\hline
\end{tabular}


pretendido inicialmente. Um exemplo é "o resultado foi menor que o pretendido no objetivo... Porém, as necessidades da empresa $A$ foram satisfeitas plenamente... tanto que a ferramenta é utilizada com sucesso na atualidade".

\subsubsection{FCSs na percepção do participante-observador}

0 projeto $\mathrm{B}$ teve duração de poucos meses, e a atuação do pesquisador foi menor que no projeto anterior. Além disso, as atividades ocorreram mais intensamente na empresa A e não no laboratório da universidade; a base de trabalho do participanteobservador e as observações coincidiram com as percepções dos integrantes até o momento apresentadas, portanto, não se faz necessário apresentá-las.

\subsubsection{Percepção dos coordenadores quanto aos resultados}

Nas respostas da parte 111 do roteiro de entrevistas, dirigido aos coordenadores do Programa U-EA e aos coordenadores de projeto, obtiveram-se respostas afirmativas de três, entre quatro respondentes, para as seguintes dimensões: benefícios financeiros e contribuição ao apoio continuado do programa de pesquisa; e todos responderam positivamente para as dimensões: envolvimento de projetos de estudantes e recrutamento de estudantes. Foram dadas respostas negativas por três de quatro respondentes para a dimensão inovação tecnológica e um respondeu que houve benefício para a empresa.

Para a dimensão publicação de artigos, ocorreu que três respondentes declararam que as metas não foram atingidas. Um relativizou, afirmando haver sido produzida uma dissertação de mestrado, ainda sem a publicação do artigo.

Em relação à opinião sobre o sucesso do projeto $\mathrm{B}$, todos os respondentes consideraram o projeto um sucesso. 0 participante-observador teve, em linhas gerais, as mesmas percepções dos coordenadores do projeto. Assim, pode-se caracterizar o projeto B, segundo suas metas, como um caso de sucesso.

\subsection{Comparação dos resultados dos projetos}

$\mathrm{Na}$ análise do ponto de vista dos participantes da equipe, sobre a presença e a influência dos FCSs (as questões fechadas do roteiro de entrevistas), verificou-se que a quantidade de fatores considerados presentes divergiu entre os projetos. No projeto A, quatro FCSs foram percebidos como presentes e influentes, e para o projeto B foram treze FCSs. 0 corte, porém, reflete que, de fato, um número menor de fatores é crítico (dos quarenta pesquisados), nos dois projetos em estudo. No entanto, não foi possível extrair uma classificação de importância devido à limitação da análise.

Uma hipótese para o ocorrido é a grande divergência nas opiniões dos membros da equipe do projeto A, uma vez que foi constatado um número de resultados conflitantes de 24 (ou seja, $60 \%$ FCSs perguntados) na verificação da presença e influência dos FCSs para o projeto A e dezesseis (40\%) para o projeto B.

Outra consideração é que há diferença na concentração dos FCSs em seus temas para os resultados tidos como presentes, ausentes ou conflitantes. A Tabela 2 demonstra a afirmação.

É possivel notar na Tabela 2 que a presença nos resultados do projeto B contém fatores críticos em quase todos os temas. É representativo, inclusive, no primeiro grupo de temas, referentes ao gerenciamento de projeto e suas técnicas (ou seja, os temas gerenciamento de projeto, aspectos gerais e gerente de projeto). Provavelmente isso explica o resultado mais satisfatório que o do outro projeto.

0 projeto $A$ apresentou conflito ou ausência nesse primeiro grupo de temas. Porém, no grupo de temas referentes à análise do parceiro (ou seja, os temas avaliação do parceiro, questões da diferença cultural, garantia de igualdade e influências externas), observou-se uma situação semelhante à do projeto B. Como o segundo grupo trata de

Tabela 2. Comparação da presença dos fatores críticos conforme os temas de FCSs. Legenda.

\begin{tabular}{|c|c|c|c|c|c|c|}
\hline \multirow[t]{2}{*}{ Objetivos } & \multicolumn{3}{|c|}{ Projeto A } & \multicolumn{3}{|c|}{ Projeto B } \\
\hline & $\begin{array}{l}\text { CP } \\
(\%)\end{array}$ & $\begin{array}{c}A \\
(\%)\end{array}$ & $\begin{array}{l}P \\
(\%)\end{array}$ & $\begin{array}{l}\text { CP } \\
(\%)\end{array}$ & $\begin{array}{c}A \\
(\%)\end{array}$ & $\begin{array}{c}P \\
(\%)\end{array}$ \\
\hline Gerenciamento & 100 & 0 & 0 & 30 & 10 & 60 \\
\hline Aspectos gerais & 85 & 15 & 0 & 15 & 15 & 70 \\
\hline $\begin{array}{l}\text { Gerenciamento de } \\
\text { projetos }\end{array}$ & 80 & 20 & 0 & 80 & 0 & 20 \\
\hline $\begin{array}{l}\text { Avaliação do } \\
\text { parceiro }\end{array}$ & 20 & 10 & 70 & 20 & 10 & 70 \\
\hline Diferencial cultural & 0 & 0 & 100 & 25 & 0 & 75 \\
\hline $\begin{array}{l}\text { Garantia de } \\
\text { igualdade }\end{array}$ & 35 & 30 & 35 & 100 & 0 & 0 \\
\hline Influências externas & 50 & 0 & 50 & 100 & 0 & 0 \\
\hline Total & 60 & 10 & 30 & 40 & 5 & 55 \\
\hline
\end{tabular}

CP: conflito para a presença; A: ausência e P: presença. 
temas mais dirigidos à relação entre os parceiros, 0 resultado era esperado, uma vez que estes, nos dois projetos, são os mesmos, contemplando a mesma trajetória, histórico e experiências, inclusive equipes com parte dos membros comuns.

0 resultado do projeto $A$ foi considerado de não sucesso, segundo a teoria de gestão de projetos, visto que não atingiu os objetivos pretendidos. Isso sugere a hipótese de que os FCSs do contexto da parceria seriam qualificadores e, havendo a sua presença, estariam criadas as condições para o estabelecimento e a continuidade dos projetos. 0 que explicaria o fato de, mesmo com tantos problemas, o projeto A ter tido continuidade e obtido algum resultado, embora diferente do esperado.

A comparação entre os dois projetos ajuda a entender melhor, também, a questão dos objetivos do projeto. A pesquisa de campo e documental indicou que as práticas quanto à definição dos objetivos são similares. Porém, no caso do projeto com maior grau de inovação tecnológica, eles não foram considerados claros desde o início, do ponto de vista dos participantes. Relatou-se ambiguidade - respostas indicando que o objetivo estava definido contrastavam com afirmações de que não estava. Essa percepção pode estar relacionada, de fato, com a diferença entre objetivos para a execução de tarefas específicas e objetivos conforme a visão do projeto.

No projeto B, a colaboração com foco no resultado principal da empresa, havia menor inovação e, portanto, a prática de definição de objetivos utilizada (descrição textual e conversas em reuniões) pode ter sido suficiente para orientar os trabalhos das equipes. No projeto A, com maior nível de inovação, porém, a mesma prática foi insuficiente. Indica-se, assim, a fonte da ambiguidade. Existia uma definição, respaldando a percepção afirmativa em determinados momentos, porém ela não foi suficiente, gerando a percepção de que não havia uma definição clara dos objetivos.

Portanto, esse fato gera a hipótese de que faltou, no projeto $\mathrm{A}$, um trabalho mais efetivo de descrição da visão e de seu desdobramento em objetivos específicos, bem como o acompanhamento da percepção dos membros do time quanto às metas especificas e interfaces com os trabalhos das demais equipes.

Outra consideração é que, tal qual apontado na literatura, as questões das diferenças culturais e a confiança foram importantes para a realização dos projetos. De fato, os fatores culturais, foco de grande número de estudos na literatura, são reconhecidos como um dos maiores obstáculos para o sucesso de projetos colaborativos universidadeempresa (BARNES; PASHBY; GIBBONS, 2002; DAVENPORT; DAVIES; GRIMES, 1998). No caso desses projetos colaborativos, da universidade com a empresa $A$, verificou-se que eles tiveram uma influência positiva em ambos os projetos. A explicação é que os membros do parceiro-empresa eram conhecedores das diferenças culturais, compreensivos e experientes em projetos de pesquisa e desenvolvimento de software com a universidade. Historicamente, possuíam também reputação no meio acadêmico da universidade em questão.

0 outro fator citado na literatura (RYCROFT; KASH, 2004; DAVIS; KEYS; CHEN, 2004; NOOTEBOOM, 2004) como crítico em projetos colaborativos U-E, e que despertou atenção nessa pesquisa, foi a confiança. Segundo Davenport, Davies e Grimes (1998), a confiança pode levar anos para ser conseguida e, uma vez estabelecida entre os parceiros, proporciona novos projetos colaborativos, nos quais mais recursos são aplicados e menores são os custos envolvidos.

Nos projetos estudados, verificou-se que a relação de confiança entre os parceiros era significativamente forte, constatada pela unanimidade da avaliação. Além das questões fechadas, essa afirmação esteve presente nos comentários da pesquisa de campo e observações feitas pelo pesquisador no decorrer dos projetos. 0 estudo reforça, portanto, a importância desse aspecto, fundamental, principalmente no projeto $\mathrm{A}$, para que os problemas fossem contornados e parte dos objetivos iniciais fosse atingida. A confiança foi construída principalmente pela atuação dos coordenadores e está mais relacionada ao contexto geral da colaboração do que a um projeto específico. Isto é, uma vez constituída, permeia os vários projetos realizados entre os parceiros.

\section{Conclusões}

0 fato de a pesquisa ter abordagem qualitativa permitiu levantar uma série de questões sobre os fatores de gerenciamento dos projetos colaborativos universidade-empresa. Especialmente pelo fato de 0 estudo ter confrontado as percepções dos envolvidos com dados observados diretamente no campo.

0 ponto mais interessante do caso é o fato de os aspectos relacionados ao contexto da parceria (identificação de parceiros, diferença cultural, igualdades e influências externas) terem sido caracterizados como presentes em ambos os projetos, mesmo havendo resultados distintos: um de sucesso e outro de não sucesso. Isso implica que 
a diferença entre os projetos A e B se deu justamente nos fatores ligados aos temas práticas de gestão e gerente de projeto.

A acepção da literatura é de que um FCS precisa ser fundamental para o sucesso do projeto. 0 resultado é, portanto, evidência forte de que tais fatores não atuaram como fatores críticos de sucesso no caso analisado. Essa é a contribuição mais interessante do artigo. Demonstra que, no caso estudado, os FCSs ligados ao contexto da parceria, como identificação de parceiros, diferença cultural, igualdades e influências externas, foram qualificadores. Embora fundamentais para compor as condições essenciais do projeto colaborativo, não foram suficientes para promover um resultado positivo, não foram decisivos. Esse indício gera a hipótese de que as listas presentes na literatura devem ser revistas. Pois, se isso se confirmar, tais listas poderiam ser reescritas e ter menor quantidade de fatores críticos, complementada por fatores qualificadores.

A segunda conclusão significativa é a necessidade de se desenvolverem escalas e métricas mais sofisticadas para avaliar esse tipo de colaboração. Os dados deste estudo são prova da "fragilidade" das escalas empregadas nos levantamentos da área, que geraram as listas de fatores críticos utilizadas na investigação. 0 resultado da avaliação objetiva foi incoerente com as respostas e dados das outras fontes de análise: análises documentais e entrevistas em profundidade.

A terceira conclusão refere-se às considerações sobre a definição de objetivos de projetos inovadores, como é o caso do projeto $A$. 0 que, à primeira vista, parecia ser um FCS fácil de se identificar, mostrou-se, na prática, de difícil medição. A causa parece ser o fato de que um objetivo pode ser dito com diferentes níveis de abstração. E, no caso de projetos inovadores e colaborativos, a mensagem fica prejudicada tanto pela falta de padrões históricos a serem seguidos como pela diferença de linguagem entre os membros do projeto. Faz-se necessário um cuidado especial, envolvendo mais do que objetivos em si.

Trabalhos de pesquisa futuros são sugeridos de forma implícita nas conclusões aqui apresentadas: estudar escalas para avaliação de FCSs em projetos colaborativos, desenvolver modelos contingenciais de FCSs, com análises que estabeleçam relações entre fatores para projetos colaborativos U-E, e métodos e técnicas para o desenvolvimento de visões compartilhadas.

Finalmente, a manifestação da intenção de ambos os parceiros em continuar a realizar novos projetos dessa natureza no futuro foi um dado relevante, pois demonstrou que, mesmo tratando-se de empreendimentos complexos e com várias dificuldades, os projetos colaborativos U-E trazem benefícios. Assim, é importante que mais pesquisadores se atenham sobre esse assunto.

\section{Referências}

ALBERTIN, E. V. Avaliação de fatores críticos de sucesso no gerenciamento de projetos colaborativos universidadeempresa. São Carlos. Dissertação (Mestrado) - Universidade de São Paulo, São Carlos, 2008.

AMATO NETO, J. Redes de cooperação produtiva e clusters regionais: oportunidades para pequenas e médias empresas. São Paulo: Atlas, 2000.

BARNES, T. A.; PASHBY, I. R.; GIBBONS, A. M. Collaborative R\&D projects: a framework for effective management. ln: IEEE INTERNATIONAL CONFERENCE ON MANAGEMENT OF INNOVATION AND TECHNOLOGY, 2000, New York. Proceedings... New York: IEEE, 2000. p. 210-216. (v. 1)

Effective university-industry interaction: a multicase evaluation of collaborative R\&D projects. European Management Journal, v. 20, n. 3, p. 272-285, 2002.

Managing collaborative R\&D projects development of a practical management tool. International Journal of Project Management, v. 24, n. 5, p. 395-404, 2006.

BUSINESS \& HIGHER EDUCATION FORUM. Working together, creating knowledge: the university-industry research collaboration iniciative. Washington: American Council on Education, 2001. Disponível em:<http://www.acenet. edu/programs/bhef/>. Acesso em: 25 jun. 2007.

CAMARINHA-MATOS, L. M.; AFSARMANESH, H. Collaborative networks: a new scientific discipline. Journal of Intelligent Manufacturing, v. 16, n. 4-5, p. 439-452, 2005.

CAMARINHA-MATOS, L. M. et al. Rough reference model for collaborative networks. Portugal: UNINOVA, 2006. Disponivel em: <http://www.ve-forum.org/projects/284/ Deliverables/D52.2_Final.pdf >. Acesso em: 10 nov. 2007.

CYERT, R. M.; GOODMAN, P. S. Creating effective universityindustry alliances: an organizational learning perspective. Organizational Dynamics, v. 25, n. 4, p. 45-57, 1997.

DANE, F. C. Research methods. Belmont: Brooks, 1990.

DAVENPORT, S.; DAVIES, J. D.; GRIMES, C. Collaborative research programs: building trust from difference. Technovation, v. 19, n. 1, p. 31-40, 1998.

EMDEN, Z.; CALANTONE, R.; DROGE, C. Collaborating for new product development: selecting the partner with maximum potential to create value. Journal of Product Innovation Management, v. 23, n. 4, p. 330-341, 2006.

GIL, A. C. Métodos e técnicas de pesquisa social. 5 ed. São Paulo: Atlas, 1999.

GODOY, A. S. Introdução à pesquisa qualitativa e suas possibilidades. Revista de Administração de Empresas, v. 35, n. 2, p. 57-63, 1995.

GOLD, R. L. Roles in sociological field observation. In: MacCALL, G. J.; SIMMOSNS, J. L. (Eds.). Issues in participant observation. Reading, MA: Addison-Wesley, 1969. 
KATZ, J. S.; MARTIN, B. R. What is research collaboration? Research Policy, v. 26, n. 1, p. 1-18, 1997.

MATTESSICH, P. W.; MONSEY, B. R. Collaboration: what makes it work. A review of research literature on factors influencing successful collaboration. Saint Paul: Amherst H. Wilder, 1992.

MORA-VALENTIN, E. M.; MONTORO-SANCHES, A.; GUERRAS-MARTIN, L. A. Determining factors in the success of R\&D cooperative agreements between firms and research organizations. Research Policy, v. 33, n. 1, p. 17-40, 2003.

PESQUISA INDUSTRIAL DE INOVAÇÃO TECNOLÓGICA PINTEC. Triênio 2001-2003. Rio de Janeiro: IBGE, 2005. Disponível em:<http://www.ibge.gov.br>. Acesso em: 15 set. 2007.

PESQUISA INDUSTRIAL DE INOVAÇÃO TECNOLÓGICA PINTEC. Triênio 2003-2005. Rio de Janeiro: IBGE, 2007. Disponível em:<http://www.ibge.gov.br>. Acesso em: 10 set. 2007.

PLONSKI, G. A. Cooperação universidade-empresa: um desafio gerencial complexo. Revista de Administração da Universidade de São Paulo, v. 34, n. 4, p. 5-12, 1999.

PORTO, G. S. Decisão empresarial de desenvolvimento tecnológico por meio da cooperação empresauniversidade. Tese (Doutorado) - Universidade de São Paulo, São Paulo, 2000.

PROJECT MANAGEMENT INSTITUTE. PMBoK@ Guide: a guide to the management body of knowledge. 3 ed. Newton Square, 2004.

ROCKART, J.; BULLEN, C. A Primer on critical success factors. Cambridge: Sloan School of Management, 1981. (Center for Information Systems Research Working Paper, n. 69)

RYCROFT, R. W.; KASH, D. E. Self-organizing innovation networks: implications for globalization. Technovation, v. 24, n. 3, p. 187-197, 2004.

SEGATTO-MENDES, A. P. Análise do processo de cooperação tecnológica universidade-empresa: um estudo exploratório. Dissertação (Mestrado) - Universidade de São Paulo, São Paulo, 1996.

VERZUH, E. MBA compacto, gestão de projetos. $11 \mathrm{ed}$. Rio de Janeiro: Elsevier, 2000.

\title{
The context of the partnership as a qualifier of university-enterprise project management
}

\begin{abstract}
There is a set of Critical Success Factors (CSF) for project management in the university-enterprise collaboration literature. A review indicated a need for new studies, applying more in-depth research methods. This paper analyzes two different projects, developed through the collaboration between a research group and a software enterprise. The analysis encompasses project coordinator and team member interviews and field diaries, which were complemented by analysis of documents. The results indicated that factors related to the management practices were more critical to the project success. The context of the university-enterprise partnership factors alone acted as qualifiers of the collaboration.
\end{abstract}

\section{Keywords}

Management of university-enterprise collaborative projects. Critical success factors. Product development. Project management. 